\title{
The Interfacial Orientation Relationship of Oxide Nanoparticles in a Hafnium-containing Oxide Dispersion-Strengthened Austenitic Stainless Steel
}

\author{
Yinbin Miao ${ }^{\mathrm{a}, *}$, Kun $\mathrm{Mo}^{\mathrm{b}}$, Bai Cui ${ }^{\mathrm{c}, \mathrm{d}}$, Wei-Ying Chen ${ }^{\mathrm{a}}$, Michael K. Miller ${ }^{\mathrm{e}}$, \\ Kathy A. Powers ${ }^{\mathrm{e}}$, Virginia McCreary ${ }^{\mathrm{d}}$, David Gross ${ }^{\mathrm{d}}$, Jonathan Almer ${ }^{\mathrm{f}}$, \\ Ian M. Robertson ${ }^{\mathrm{g}, \mathrm{d}}$, James F. Stubbins ${ }^{\mathrm{a}}$
${ }^{a}$ Department of Nuclear, Plasma and Radiological Engineering, University of Illinois at Urbana-Champaign, Urbana, IL 61801, USA
${ }^{b}$ Nuclear Engineering Division, Argonne National Laboratory, Lemont, IL 60493, USA
${ }^{c}$ Department of Mechanical and Materials Engineering, University of Nebraska-Lincoln, Lincoln, NE 68588
${ }^{d}$ Department of Material Science and Engineering, University of Illinois at Urbana-Champaign, Urbana, IL 61801, USA
${ }^{e}$ Center for Nanophase Materials Sciences, Oak Ridge National Laboratory, Oak Ridge, TN, 37830, USA
${ }^{f} X$-ray Science Division, Argonne National Laboratory, Lemont, IL 60493, USA
${ }^{g}$ Department of Material Science and Engineering, University of Wisconsin-Madison, Madison, WA,53706, USA

\begin{abstract}
This work reports comprehensive investigations on the orientation relationship of the oxide nanoparticles in a hafnium-containing austenitic oxide dispersion-strengthened 316 stainless steel. The phases of the oxide nanoparticles were determined by a combination of scanning transmission electron microscopy - electron dispersive X-ray spectroscopy, atom probe tomography and synchrotron X-ray diffraction to be complex Y-Ti-Hf-O compounds with similar crystal structures, including bixbyite $\mathrm{Y}_{2} \mathrm{O}_{3}$, fluorite $\mathrm{Y}_{2} \mathrm{O}_{3}-\mathrm{HfO}_{2}$ solid solution and pyrochlore (or fluorite) $\mathrm{Y}_{2}(\mathrm{Ti}, \mathrm{Hf})_{2-x} \mathrm{O}_{7-2 x}$. High resolution transmission electron microscopy was used to characterize the particle-matrix interfaces. Two different coherency relationships along with one axis-parallel relation between the oxide nanoparticles and the steel matrix were found. The size of the nanoparticles significantly influences the orientation relationship. The results provide insight into the relationship of these nanoparticles with the matrix, which has implications for interpreting material properties as well as responses to radiation.
\end{abstract}

Keywords: structure materials, ODS, atom probe tomography, TEM, synchrotron diffraction, orientation relationship

\section{Introduction}

Oxide dispersion-strengthened (ODS) alloys, due to their outstanding creep resistance and radiation tolerance $[1,2]$, are promising candidate materials for structural components in prospective

\footnotetext{
*Address: 216 Talbot Laboratory, 104 South Wright Street, Urbana, IL 61801 Tel: 217-607-6294 Email: miao2@illinois.edu

Preprint submitted to Materials Characterization

January 20, 2015
} 
energy systems including advanced fission and fusion reactors. The development of ODS alloys has primarily focused on ferritic or martensitic steels due to their advantages in mechanical strength $[3,4]$. However, austenitic steels have superior corrosion resistance to ferritic steels, and so have a greater potential for use in higher temperature applications. Hence, a series of austenitic ODS stainless steels[5, 6, 7], including a hafnium-containing ODS 316 steel[8], were produced in order to enhance the corrosion resistance with little compromise in mechanical strength and radiation tolerance, and to extend the application to higher temperature service conditionsl.

In ODS alloys, the interfaces between the oxide nanoparticles and the matrix significantly influence material performance. In particular, the challenge is to understand the fundamental physical processes responsible for stabilization of the oxygen-enriched nanoparticles to improve high-temperature strength and creep deformation performance. The orientation between the nanoparticles and the matrix determines the type of the nanoparticle-dislocation interaction, which is directly related to the strengthening mechanisms[9]. Also, the interfaces of dispersive nanoparticles can provide a great number of recombination centers for point defects[2, 8]. In addition to point defect annihilation, helium atoms can be trapped by these interfaces to relieve irradiation-induced embrittlement or mitigate the tendency for helium to support void or bubble nucleation[10, 11, 12, 13]. These phenomena are driven by the elastic interaction of the stress fields due to the matrix-nanoparticle interfaces and the various point or line defect structures[14, 15]. The character of the matrix-nanoparticle interface is, in turn, controlled by the orientation relationship. Multiple advanced investigation methods have been employed to characterize these orientation relationships in ferritic ODS steels[16, 17, 18, 19]. A recent high resolution transmission electron microscopy (HR-TEM) investigation in ODS 316 stainless steel discovered the existence of the cubic-on-cubic relation[20]. However, more complex chemical composition and orientation relationships, especially those related to the coherency mechanisms, are expected in the ODS 316 stainless steel and not covered by previous studies. In addition, the size effect of the nanoparticles on the orientation relationship is required to better understand the behavior of the nanoparticles as well as to guide the development of future austenitic ODS alloys.

The scanning transmission electron microscopy (STEM), combined with spectroscopies, such as electron dispersive X-ray spectroscopy (EDS) or electron energy loss spectroscopy (EELS), is powerful for the characterization of dispersed inclusion phases with diameters around 5 to 100 $\mathrm{nm}[21,22,23]$. On the other hand, atom probe tomography (APT) was successfully utilized to analyze the oxygen-enriched nanoclusters with extremely small size $(<5 \mathrm{~nm})$ in ODS steels[24]. Therefore, the combination of STEM and APT techniques provide a method for chemical composition investigations that are capable of covering inclusions with a wide range of dimensions. In addition, the high energies and high intensities of $\mathrm{X}$-rays produced by synchrotron radiation provide the opportunity to collect diffraction data from inclusion phases of low volume fractions, such as the oxygen-enriched nanoparticles in ODS steels, a task which is impossible with conventional $\mathrm{X}$-ray sources. This paper reports the chemical composition and structure characterization by the joint efforts of STEM, APT and synchrotron X-ray diffraction (XRD) followed by an HR-TEM investigation of ODS nanoparticle size influence on the orientation relationship of oxide nanoparticles within a 316 stainless steel matrix.

\section{Description of Experiments}

The SUS316 stainless steel, Fe-16.16Cr-13.66Ni-2.33Mo-1.82Mn-0.08Ti-0.75Si-0.08(Nb+Ta) $-0.05 \mathrm{C}$ by wt $\%$, was ball milled with $0.35 \mathrm{wt} \% \mathrm{Y}_{2} \mathrm{O}_{3}, 0.1 \mathrm{wt} \% \mathrm{Ti}$ and $0.6 \mathrm{wt} \% \mathrm{Hf}$ for 24 hours. The product was annealed at $1150{ }^{\circ} \mathrm{C}$ for 2 hours before hot extrusion, and was finally annealed 
at $1100{ }^{\circ} \mathrm{C}$ for 1 hour. The material was manufactured by Prof. Somei Ohnuki's research group at Hokkaido University[20]. The grain size of the ODS alloy 316 is approximately $0.5 \mu \mathrm{m}$, and the oxide particle density is $6.6 \times 10^{21} \mathrm{~m}^{-3}$ with an average size of $9.4 \mathrm{~nm}$ according to the manufacturer. The alloy was mechanically polished to $100 \mu \mathrm{m}$ and then punched into $3 \mathrm{~mm}$ TEM discs. Finally, the alloy was thinned to electron transparency by electropolishing with a solution containing $5 \mathrm{v} \%$ perchloric acid and $95 \mathrm{v} \%$ methanol at $-14{ }^{\circ} \mathrm{C}$.

The APT specimens were fabricated from in-situ lift-out specimens extracted from the surface of the electropolished $3 \mathrm{~mm}$ TEM disc specimen and then annular milled in a Dualbeam FEI Nova 200 Nanolab focused ion beam/scanning electron microscope (FIB/SEM).[25]. APT characterization was performed in an energy-compensated CAMECA Instruments Inc. local electrode atom probe (LEAP ${ }^{\circledR} 4000 X$ HR). Due to the poor electrical and thermal conduction of these materials, the specimens were analyzed in laser-mode at a specimen temperature of $30 \mathrm{~K}$, a pulse repetition rate of $200 \mathrm{kHz}$, a focused laser beam energy of either 50 or $100 \mathrm{pJ}$, and a data collection rate between $0.5 \%$ and $4 \%$ ions per field evaporation pulse depending on the standing voltage applied to the specimen. The position of the laser beam on the apex of the specimen was adjusted automatically during the experiment to account for the field evaporation of material from the apex of the specimen and specimen drift. These conditions resulted in individual LEAP ${ }^{\circledR}$ datasets containing up to 200 million atoms. Surface regions that contained damage from the gallium ion beam were not used for analysis. Data analysis was performed with the use of CAMECA Instruments Inc. Integrated Visualization and Analysis Software (IVAS 3.6.6). The proxigrams[26] were calculated according to the isosurfaces defined by $8 \%$ decomposed oxygen concentration. Thus, the full width at half maximum (FWHM) of the oxygen concentration in proxigrams was regarded as the size of the oxygen-enriched nanoclusters.

The TEM samples were characterized by using the STEM high angle annual dark field (HAADF) Z-contrast technique to locate the oxide nanoparticles. In addition, EDS analysis was used to characterize the chemical composition of these nanoparticles. The STEM-EDS characterization was conducted using a JEOL 2010F EF-FEG (energy filtered and field emission gun) TEM. The HR-TEM method was used to determine the orientation relationship between the oxide nanoparticles and the steel matrix. The HR-TEM was conducted on a JEOL 2010 $\mathrm{LaB}_{6}$ TEM operated at $200 \mathrm{kV}$. In addition, the bulk ODS 316 steel specimen was investigated by wide angle X-ray scattering (WAXS) using $86 \mathrm{keV}$ synchrotron photons in Sector 1-ID at the Advanced Photon Source (APS), Argonne National Laboratory (ANL).

\section{Results and Discussion}

The STEM HAADF image, Fig. 1(a), illustrated the prominent $Z$-contrast of the oxide nanoparticles. EDS indicates that the particles are complex oxides containing Y, Ti, and Hf (see Fig. 2). Due to the existence of the heavy element Hf, the contrast of the nanoparticles appears brighter than those of Y-Ti-O nanoparticles observed in other ODS alloys. APT data reconstruction also indicated that the oxygen-enriched dispersed nanoparticles smaller than $5 \mathrm{~nm}$ (also called nanoclusters) contain Y, Ti and $\mathrm{Hf}$ (Fig. 3). The quantitative analysis results of the oxygen-enriched nanoparticles by both STEM-EDS and APT are illustrated in a ternary coordinate system so that the contents of all three major metal elements (Y, Ti, and Hf) can be shown in Fig. 4. The color of the data points indicates the size of the oxygen-enriched nanoparticles obtained by measuring STEM images or APT proxigram data. According to these chemical composition data, the Y-Ti-Hf-O inclusions can be divided into three discrete groups: $\mathrm{Y}_{2} \mathrm{O}_{3}, \mathrm{Y}_{2} \mathrm{O}_{3}-\mathrm{HfO}_{2}$ solid solution, and $\mathrm{Y}_{2}(\mathrm{Ti}, \mathrm{Hf})_{2-x} \mathrm{O}_{7-2 x} . \mathrm{Y}_{2} \mathrm{O}_{3}$, which has a bixbyite structure, was present in 
the original oxide powders added before ball milling. It is well known that the $\mathrm{Y}_{2} \mathrm{O}_{3}$ and $\mathrm{HfO}_{2}$ can form solid solution with fluorite structure[27], and that the $\mathrm{Y}_{2} \mathrm{O}_{3}-\mathrm{HfO}_{2}$ solid solution has a minimum enthalpy near $\mathrm{Y} / \mathrm{Hf}=1$, namely, $\mathrm{Y}_{2} \mathrm{Hf}_{2} \mathrm{O}_{7}$ [28]. It had been believed until recently that $\mathrm{Y}_{2} \mathrm{Ti}_{2} \mathrm{O}_{7}$ was the only cubic phase Y-Ti-O compound, whereas another common Y-Ti-O compound, $\mathrm{Y}_{2} \mathrm{TiO}_{5}$ had an orthorhombic structure. According to recent reports[29], $\mathrm{Ti}$ atoms in $\mathrm{Y}_{2} \mathrm{Ti}_{2} \mathrm{O}_{7}$ could be replaced by $\mathrm{Y}$ through vacancy compensation mechanism[30] so that pyrochlore $\mathrm{Y}_{2} \mathrm{Ti}_{2-x} \mathrm{O}_{7-2 x}$, including $\mathrm{Y}_{2} \mathrm{TiO}_{5}$, could form[31]. As Ti and $\mathrm{Hf}$ belong to the same group on the periodic table, it is plausible that $\mathrm{Hf}$ can replace $\mathrm{Ti}$ in $\mathrm{Y}_{2} \mathrm{Ti}_{2-x} \mathrm{O}_{7-2 x}$. Thus, $\mathrm{Y}_{2}(\mathrm{Ti}, \mathrm{Hf})_{2-x} \mathrm{O}_{7-2 x}$ whould have a pyrochlore or fluorite structure, depending on the $\mathrm{Hf} / \mathrm{Ti}$ ratio. For example, the boundary ratio of $\mathrm{Hf} / \mathrm{Ti}$ between fluorite and pyrochlore structures for $\mathrm{Y}_{2}(\mathrm{Ti}, \mathrm{Hf})_{2} \mathrm{O}_{7}$ is $\mathrm{Hf} / \mathrm{Ti}=7.71$ according to the averaged ion radius[32] and the judging criteria in Ref. [20]. An interesting phenomenon involving $\mathrm{Y}_{2}(\mathrm{Ti}, \mathrm{Hf})_{2-x} \mathrm{O}_{7-2 x}$ nanoparticles is that the Z-contrast of these particles actually reflects their Hf/Ti ratio. The HAADF-STEM images of $\mathrm{Y}_{2}(\mathrm{Ti}, \mathrm{Hf})_{2-x} \mathrm{O}_{7-2 x}$ nanoparticles, Fig. 1(b) through (e), illustrate the variation in the Hf/Ti ratios measured by quantitative EDS analyses.

Because both bixbyite and pyrochlore structures are variants of the fluorite structure, all have similar major diffraction peaks. Therefore, synchrotron XRD data can only show the oxide peaks ( $\{222\}$ and $\{400\})$ to which all three inclusion phases mentioned above contribute (see Fig. 5). Due to the existence of extra oxygen vacancies in both bixbyite and pyrochlore structures, the atoms in these two structures are displaced from the reference fluorite lattice positions, making the spatial periods twice that of the fluorite structure. Therefore, the Miller indices of these two variants are different by a factor of 2 from their equivalents of the fluorite structure. In this manuscript, all these values are expressed according to the pyrochlore or bixbyite structure regardless of the actual structure of the nanoparticle for uniformity. The lattice parameter of the austenite matrix was determined to be $3.595 \AA$.

Most of the nanoparticles are too small to use electron diffraction to determine the orientation directly, so the fast Fourier transformation (FFT) results of the HR-TEM images of these nanoparticles were utilized to determine the electron diffraction patterns and lattice spacings. The electron diffraction information ( $\{222\},\{400\}$, and $\{440\}$ diffraction spots) indicates that the nanoparticles have a defective fluorite structure or its derivatives. These electron diffraction results are consistent with the synchrotron scattering data, as the two synchrotron-distinguishable peaks $(\{222\}$ and $\{400\}$ ) corresponds to the strongest reflection of the fluorite-related structures. Due to the similarity of bixbyite, pyrochlore, and fluorite structures, it was difficult to distinguish them in HR-TEM images. Fortunately, this similarity also could imply that these nanoparticles should have similar orientation relationships with the matrix. All three groups of nanoparticles were analyzed together in the discussion of the orientation relationship.

The size distribution of these oxide nanoparticles was determined from direct measurement of individual particles in the TEM images. Although there are occasional exceptions, the overwhelming majority of the oxide particles have diameters smaller than $30 \mathrm{~nm}$. It is worth mentioning that the extremely small nanoclusters $(<2 \mathrm{~nm})$ were not distinguishable in TEM observations[23]. A size distribution of 110 randomly-selected oxide nanoparticles is shown in Fig. 6(a). The average diameter is $10.9 \pm 1.2 \mathrm{~nm}$ according to the measurement, consistent with the manufacturer's data.

Electron diffraction information from HR-TEM of both the matrix and the nanoparticles indicated the existence of two coherency mechanisms of the nanoparticles. The HREM image along with its FFT diffraction pattern, Fig. 7, illustrates one type of coherency mechanisms (labeled as Coherency I). Here, the $[110]_{o x}$ axis and the $[001]_{\text {mat }}$ axis, which are the zone axes in Fig. 7, are parallel. Also, the $(\overline{4} 40)_{o x}$ planes are parallel to the $(200)_{m a t}$ planes, according 
to the overlapped diffraction spots in the FFT pattern (Fig. 7(c)). The other type of coherency mechanism (labeled as Coherency II) is shown in Fig. 8. Here, the zone axes of both the particle and the matrix are [110] with overlapping $(\overline{4} 40)_{o x}$ and (002) mat spots. The spots in Fig. 7(c) and (d) labeled by "MF" correspond to the Moire fringes formed by the $\{020\}_{\text {mat }}$ and $\{222\}_{o x}$ reflections. Likewise, the Moiré fringes shown in Fig. 8(c) and (d) (labeled by "MF") are due to the $\{111\}_{\text {mat }}$ and $\{222\}_{o x}$ reflections. The orientation and spacing of these Moiré fringes can be predicted precisely by the following equations: $D=d_{1} d_{2} / \sqrt{d_{1}^{2}+d_{2}^{2}-2 d_{1} d_{2} \cos \alpha}$ and $\Theta=\arcsin \left(d_{1} \sin \alpha / \sqrt{d_{1}^{2}+d_{2}^{2}-2 d_{1} d_{2} \cos \alpha}\right)$; where $d_{1}$ and $d_{2}$ are the d-spacings of the two atomic fringes; $\alpha$ is the angle between them; $\mathrm{D}$ is the spacing of the double diffraction fringes; and $\Theta$ is the angle between $d_{1}$ and $\mathrm{D}$.

For the condition [110] mat $\|[110]_{o x}$, the $(00 \overline{2})_{\text {mat }}$ and $(\overline{4} 40)_{o x}$ are not always necessarily parallel. In the case that they are not, the nanoparticle and the matrix lack a pair of parallel planes with similar d-spacings, which makes this orientation relationship unqualified for a coherency mechanism. Typically, the $[110]_{\text {mat }} \|[110]_{o x}$ axis-parallel also prefers to have one or two pairs of extra $\langle 111\rangle_{\text {mat }} \|\langle 111\rangle_{o x}$ parallel axes, which are shown in Fig. 9(a), (b) (labeled as Axis-Parallel I) and (e), (f) (labeled as Axis-Parallel II). When two pairs of $\langle 111\rangle$ axes are parallel, the cubic-on-cubic relation is formed. For all the axis-parallel cases, there exist no parallel planes with close d-spacings. In addition, all the Moiré fringe spots (labeled "MF") can be predicted by the equations given above.

One hundred and ten randomly-selected oxide nanoparticles were categorized into four groups: Coherency I, Coherency II, axis-parallel, and random orientation versus their sizes. Statistical results are shown in Fig. 6(b). The smaller the oxide particles, the larger proportion that have a non-random orientation relationship with the matrix. When the particles are smaller than $4 \mathrm{~nm}$, all exhibit a specific orientation relationship with the matrix. As the particle size increases to $20 \mathrm{~nm}$, the probability of coherency or the axis-parallel relation becomes marginal. A typical large nanoparticle $(32 \mathrm{~nm})$ with random orientation is illustrated in Fig. 10. Among the three non-random orientation relationships, the axis-parallel relation always has the highest probability of existence, whereas Coherency I has the lowest.

The lattice constant of the strain-free Y-Ti-Hf-O nanoparticles, $\mathrm{Y}_{2} \mathrm{Hf}_{2} \mathrm{O}_{7}, \mathrm{Y}_{2} \mathrm{Ti}_{2} \mathrm{O}_{7}, \mathrm{Y}_{2} \mathrm{O}_{3}$, $\mathrm{HfO}_{2}$, and $\mathrm{Y}_{2} \mathrm{TiO}_{5}$, are shown in Table 1. The information of more complex oxide phases, such as $\mathrm{Y}_{2} \mathrm{O}_{3}-\mathrm{HfO}_{2}$ solid solution and $\mathrm{Y}_{2}(\mathrm{Ti}, \mathrm{Hf})_{2-x} \mathrm{O}_{7-2 x}$, can also be assessed referring to Table 1. Due to the different chemical composition groups and various $\mathrm{Hf} / \mathrm{Ti}$ values, the (equivalent pyrochlore) lattice constants could vary from $10.090 \AA$ to $10.600 \AA$, which explains the abnormal broadening of the Y-Ti-Hf-O peaks in Fig. 5, which can not be explained merely by the size broadening. Several similar d-spacings were found between the oxide nanoparticle and the steel matrix. $d_{200}^{\text {mat }}=1.798 \AA$ is close to $d_{440}^{o x}=1.784 \sim 1.874 \AA$, whereas $d_{110}^{\text {mat }}=2.542 \AA$ is similar to $d_{400}^{o x}=2.523 \sim 2.650 \AA$. Thus, Coherency I actually includes four sets of parallel planes $\left((200)_{\text {mat }}\right.$ \| $\left.(440)_{o x} ;(101)_{\text {mat }}\left\|(040)_{o x} ;(\overline{1} 01)_{\text {mat }}\right\|(400)_{o x} ;(002)_{\text {mat }} \|(440)_{o x}\right)$ whereas Coherency II includes two sets of parallel planes $\left((002)_{\text {mat }}\left\|(\overline{4} 40)_{o x} ;(1 \overline{1} 0)_{\text {mat }}\right\|(004)_{o x}\right)$. The axis-parallel relation does not involve any parallel planes with close d-spacings. For the special cases of axis-parallel, Axis-Parallel I is a cubic-on-cubic relation which has been reported by Oka et al. [20]. However, the misfits $(\delta)$ in all parallel d-spacings are as large as 0.4, which implies the incoherent orientation relation and the dense misfit dislocations as shown in Fig. 9 (d). When only one $\langle 111\rangle_{\text {mat }} \|\langle 111\rangle_{o x}$ relation exists (Axis-Parallel II), the particles may suffer a large asymmetric strain field due to the large misfit, showing distorted FFT diffraction patterns, 
Fig. 9(f).

Assuming identical elastic moduli for the matrix and the inclusion, the free energy of a randomly-oriented spherical nanoparticle only contains the interface energy, $4 \pi r^{2} \gamma_{r}$. The formation of coherent orientation relationships reduce the areal interface energy to $4 \pi r^{2} \gamma_{c}$ by introducing an additional volumetric strain energy, $\left(4 \pi r^{3} / 3\right) \cdot 4 \mu \delta^{2}$ [33]. Here, $r$ is the nanoparticle radius; $\gamma_{r}$ and $\gamma_{c}$ are the unit areal energies of randomly-oriented and coherent interfaces, respectively, $\gamma_{r}>\gamma_{c} ; \mu$ is the shear modulus and $\delta$ is the misfit. A similar phenomenon occurs in the axis-parallel relation when misfit dislocations form on the interface, lowering the interface energy by accommodating the strain energy. Actually, the elastic moduli of the matrix and the nanoparticles usually differ a lot, but the fundamental physics of the competition between the interface energy and the strain energy remains the same. Hence, thermodynamically, the coherency and the axis-parallel relations are only favored for small nanoparticles, whereas random orientations are preferred for large nanoparticles.

The formation mechanism of oxygen-enriched precipitates in ODS steels has been studied for years. It is widely believed that the originally added $\mathrm{Y}_{2} \mathrm{O}_{3}$ powders are dissolved during ball milling and that the oxygen-enriched nanoparticles precipitate during the post-milling annealing procedure [34, 35, 36, 37]. According to Ref. [34], the precipitation initiates from the amorphous oxygen-enriched regions formed during ball milling. The formation of these amorphous regions is actually a consequence of the diffusion of dissolved $\mathrm{Y}_{2} \mathrm{O}_{3}$ as well as those metal elements that have high affinity for oxygen such as Ti and $\mathrm{Hf}$. In bcc ferrite, these amorphous regions may simply be a disordered structure formed due to the energy preference of $\mathrm{O}_{i}$-vacancy pairs in the presence of Ti[38]. The size and chemical composition evolution of the amorphous regions is therefore driven by the chemical potential and also kinetically limited by local distribution of $\mathrm{Y}, \mathrm{Ti}, \mathrm{Hf}$, and $\mathrm{O}$ atoms. The properties of these amorphous regions then determine the size and chemical composition of nanoparticles crystalized during annealing. The addition of Hf may increase the complexity of the formation of amorphous regions, which results in the existence of three separate oxygen-enriched phases. The chemical compositions of extremely small nanoclusters are frequently deviated from stoichimetric, causing the difficulties in crystallization[39]. Otherwise, for small amorphous regions, the coherent or axis-parallel orientation relation is selected during the crystallization process due to the preference in energy. Meanwhile, large randomly-orientated nanoparticles are formed to avoid the high strain energy due to strong interfacial coupling. No prominent shell structure was found in these nanoparticles.

Although Coherency I involves four pairs of parallel planes and Coherency II involves only two pairs, they should have the same elastic energy if both the matrix and the inclusion phases have isotropic elastic properties. However, both austenite and complex Y-Ti-Hf-O phases have anisotropic elastic properties (e.g., Zener's elastic anisotropy constant $\mathrm{A}_{Y_{2} T i_{2} O_{7}}=2.11, \mathrm{~A}_{S S 316}=3.78$ )[40, 41]. Therefore, Coherencies I and II have different strain energies, which can explain why Coherency II was observed more frequently than Coherency I. In addition, the variation of the nanoparticles' lattice constants due to the diverse chemical compositions influences the strain field of the coherent nanoparticles. This factor should also be considered in the explanation of the relative ratio of different orientation relations.

The small oxide nanoparticles in ODS 316 stainless steel were observed to have a significant probability of holding coherency or axis-parallel orientation relations with the matrix. Hence, the stress fields of these nanoparticles could enhance the mechanical strength and the radiation resistance performance $[14,15]$. As the nanoparticles are the consequences of crystallization of the amorphous oxygen-enriched regions that were formed during ball milling, the properties of these nanoparticles, including size, chemical composition and orientation, are believed to 
be quite stable at elevated temperature. Differences in thermal expansion properties between the nanoparticles and the matrix could cause the stress field as well as the strain energy of the coherent/axis-parallel interfaces to vary with temperature. Therefore, the radiation resistance and the mechanical strengthening properties of ODS steels would depend highly on their operating temperature, which is worth further investigation.

\section{Conclusion}

The oxide nanoparticles in a Hf-containing ODS 316 stainless steel were determined to consist of three separate groups of oxygen-enriched phases with similar crystal structures: $\mathrm{Y}_{2} \mathrm{O}_{3}$, $\mathrm{Y}_{2} \mathrm{O}_{3}-\mathrm{HfO}_{2}$ solid solution, and $\mathrm{Y}_{2}(\mathrm{Ti}, \mathrm{Hf})_{2-x} \mathrm{O}_{7-2 x}$. Two coherent orientation mechanisms, one cubic-on-cubic orientation relationship, and the general axis-parallel relationship were all observed by HR-TEM. Statistical analyses of these categories demonstrated a strong size effect of the nanoparticles on the orientation relationship. An evolution route for the development of the various orientation relationships was developed, and both energy preference and kinetics were utilized to help understand the formation of different orientation relationships.

\section{Acknowledgement}

This work was supported by 973 DOE INL 120293. The TEM experiments were carried out in part in the Frederick Seitz Materials Research Laboratory Central Facilities, University of Illinois, which is partially supported by the U.S. Department of Energy under grants DEFG02-07ER46453 and DE-FG02-07ER46471. Atom probe tomography (APT) was conducted at the Center for Nanophase Materials Sciences, which is a DOE Office of Science User Facility. M.K.M. was sponsored by the Materials Sciences and Engineering Division, Office of Basic Energy Sciences, US Department of Energy. Argonne National Laboratory's work was supported by U.S. DOE under Contract no. DE-AC02-06CH11357. We gratefully acknowledge that the ODS316 stainless steel investigated in this study was provided by Prof. Somei Ohnuki at Hokkaido University.

\section{References}

[1] M. S. El-Genk, J.-M. Tournier, J. Nucl. Mater. 340 (2005) 93-112.

[2] G. Odette, M. Alinger, B. Wirth, Annu. Rev. Mater. Res. 38 (2008) 471-503.

[3] S. Ukai, M. Fujiwara, J. Nucl. Mater. 307 - 311, Part 1 (2002) 749 - 757.

[4] K. Mo, Z. Zhou, Y. Miao, D. Yun, H.-M. Tung, G. Zhang, W. Chen, J. Almer, J. F. Stubbins, J. Nucl. Mater. 455 (2014) $376-381$.

[5] M. Wang, Z. Zhou, H. Sun, H. Hu, S. Li, Mater. Sci. Eng. A 559 (2013) 287-292.

[6] M. Wang, Z. Zhou, Z. Yan, P. Yu, H. Sun, Acta. Metall. Sinica. 49 (2013) 153-158.

[7] Y. Miao, K. Mo, Z. Zhou, X. Liu, K.-C. Lan, G. Zhang, M. K. Miller, K. A. Powers, J. Almer, J. F. Stubbins, Mater. Sci. Eng. A 625 (2015) $146-152$.

[8] H. Oka, M. Watanabe, H. Kinoshita, T. Shibayama, N. Hashimoto, S. Ohnuki, S. Yamashita, S. Ohtsuka, J. Nucl. Mater. 417 (2011) 279-282.

[9] S. Campos, H. J. Kestenbach, E. Morales, Metall. Mater. Trans. A 32 (2001) 1245-1248.

[10] K. Yutani, H. Kishimoto, R. Kasada, A. Kimura, J. Nucl. Mater. 367 (2007) 423-427.

[11] J. Chen, P. Jung, W. Hoffelner, H. Ullmaier, Acta. Mater. 56 (2008) 250-258.

[12] Q. Li, C. Parish, K. Powers, M. Miller, J. Nucl. Mater. 445 (2014) 165-174.

[13] P. Edmondson, C. Parish, Q. Li, M. Miller, J. Nucl. Mater. 445 (2014) 84-90.

[14] W. Wolfer, M. Ashkin, J. Appl. Phys. 46 (1975) 547-557.

[15] J. Dundurs, T. Mura, J. Mech. Phys. Solid. 12 (1964) 177-189.

[16] M. Klimiankou, R. Lindau, A. Möslang, J. Cryst. Growth. 249 (2003) 381-387. 
[17] A. Hirata, T. Fujita, Y. Wen, J. Schneibel, C. Liu, M. Chen, Nat. Mater. 10 (2011) 922-926.

[18] L. Hsiung, M. Fluss, S. Tumey, J. Kuntz, B. El-Dasher, M. Wall, B. Choi, A. Kimura, F. Willaime, Y. Serruys, J. Nucl. Mater. 409 (2011) 72-79.

[19] J. Ribis, Y. De Carlan, Acta. Mater. 60 (2012) 238-252.

[20] H. Oka, M. Watanabe, N. Hashimoto, S. Ohnuki, S. Yamashita, S. Ohtsuka, J. Nucl. Mater. (2013).

[21] M. Klimiankou, R. Lindau, A. Möslang, Micron. 36 (2005) 1-8.

[22] M. C. Brandes, L. Kovarik, M. K. Miller, M. J. Mills, J. Mater. Sci. 47 (2012) 3913-3923.

[23] A. Certain, K. G. Field, T. R. Allen, M. Miller, J. Bentley, J. Busby, J. Nucl. Mater. 407 (2010) 2-9.

[24] M. K. Miller, C. M. Parish, Mater. Sci. Technol. 27 (2011) 729-734.

[25] M. K. Miller, K. F. Russell, K. Thompson, R. Alvis, D. J. Larson, Microsc. Microanal. 13 (2007) $428-436$.

[26] O. C. Hellman, J. A. Vandenbroucke, J. Rüsing, D. Isheim, D. N. Seidman, Microsc. Microanal. 6 (2000) $437-444$.

[27] E. Rauwel, C. Dubourdieu, B. Holländer, N. Rochat, F. Ducroquet, M. D. Rossell, G. Van Tendeloo, B. Pelissier, Appl. Phys. Lett. 89 (2006) 012902.

[28] T. A. Lee, A. Navrotsky, J. Mater. Res. 19 (2004) 1855-1861.

[29] M. Robinson, N. Marks, M. Qin, S. Middleburgh, G. Thorogood, G. Lumpkin, D. Carter, in: TMS 2014: Annual Meeting.

[30] Y. Miao, D. Aidhy, W.-Y. Chen, K. Mo, A. Oaks, D. Wolf, J. F. Stubbins, J. Nucl. Mater. 445 (2014) 209-217.

[31] K. R. Whittle, M. G. Blackford, R. D. Aughterson, G. R. Lumpkin, N. J. Zaluzec, Acta. Mater. 59 (2011) 7530-7537.

[32] R. Shannon, Acta Cryst. Sect. A 32 (1976) 751-767.

[33] D. A. Porter, K. E. Easterling, Phase transformations in metals and alloys, CRC PressI Llc, 1992.

[34] L. L. Hsiung, M. J. Fluss, S. J. Tumey, B. W. Choi, Y. Serruys, F. Willaime, A. Kimura, Phys. Rev. B 82 (2010) 184103.

[35] M. Brocq, B. Radiguet, S. Poissonnet, F. Cuvilly, P. Pareige, F. Legendre, J. Nucl. Mater. 409 (2011) 80-85.

[36] M. Alinger, G. Odette, D. Hoelzer, Acta. Mater. 57 (2009) 392-406.

[37] S. Yamashita*, S. Ohtsuka, N. Akasaka, S. Ukai, S. Ohnuki, Philos. Mag. Lett. 84 (2004) 525-529.

[38] M. K. Miller, C. L. Fu, M. Krcmar, D. T. Hoelzer, C. T. Liu, Front. Mater. Sci. China. 3 (2009) 9-14.

[39] M. K. Miller, C. M. Parish, Q. Li, Mater. Sci. Technol. 29 (2013) 1174-1178.

[40] J. Pruneda, E. Artacho, Phys. Rev. B 72 (2005) 085107.

[41] L. Vitos, P. A. Korzhavyi, B. Johansson, Nat. Mater. 2 (2003) 25-28.

[42] M. Tamura, H. Sakasegawa, K. Shiba, H. Tanigawa, K. Shinozuka, H. Esaka, Metall. Mater. Trans. A 42 (2011) 2176-2188.

[43] H. Fukumoto, T. Imura, Y. Osaka, Appl. Phys. Lett. 55 (1989) 360-361.

[44] R. R. Manory, T. Mori, I. Shimizu, S. Miyake, G. Kimmel, J. Vac. Sci. Technol. A 20 (2002) 549-554. 


\section{Table Captions}

Table 1: Lattice parameters of the complex oxides related to Y-Ti-Hf-O inclusion phases.

Tables

\begin{tabular}{ccccc}
\multicolumn{5}{c}{ Table 1: } \\
\hline Phase & Structure & $a_{0}(\AA)$ & equivalent pyrochlore $\mathrm{a}_{0}(\AA)$ & Reference \\
\hline \hline $\mathrm{Y}_{2} \mathrm{Hf}_{2} \mathrm{O}_{7}$ & fluorite & 5.202 & 10.404 & {$[20]$} \\
$\mathrm{Y}_{2} \mathrm{Ti}_{2} \mathrm{O}_{7}$ & pyrochlore & 10.090 & 10.090 & {$[42]$} \\
$\mathrm{Y}_{2} \mathrm{O}_{3}$ & bixbyite & 10.600 & 10.600 & {$[43]$} \\
$\mathrm{HfO}_{2}$ & fluorite & 5.120 & 10.240 & {$[44]$} \\
$\mathrm{Y}_{2} \mathrm{TiO}_{5}$ & pyrochlore & 10.256 & 10.256 & {$[31]$} \\
\hline
\end{tabular}




\section{Figure Captions}

Figure 1: STEM HAADF images: (a) typical microstructure of ODS 316 stainless steel; (b) through (e) $\mathrm{Y}_{2}(\mathrm{Ti}, \mathrm{Hf})_{2-x} \mathrm{O}_{7-2 x}$ nanoparticles with various $\mathrm{Hf} / \mathrm{Ti}$ ratios. The discrete $\mathrm{Hf} / \mathrm{Ti}$ ratios were selected only to show the significant variation of this quantity.

Figure 2: A typical EDS result of an oxide nanoparticle showing the enrichment of Y, Ti, Hf, and O.

Figure 3: Atom probe tomography data showing the enrichment of $\mathrm{Y}, \mathrm{Ti}, \mathrm{Hf}$, and $\mathrm{O}$ in nanoclusters: $\mathrm{Y}, \mathrm{Ti}$, and $\mathrm{Hf}$ are enriched in these nanoclusters due to their strong affinity for oxygen.

Figure 4: Chemical composition distribution of oxide particles with various dimensions and compositions in ODS 316 steel (circles from STEM-EDS and diamonds from APT).

Figure 5: Synchrotron X-ray diffraction of ODS 316.

Figure 6: Nanoparticle size and orientation relationship: (a) normalized size distribution of oxide nanoparticles; (b) size dependency of the orientation relationship of nanoparticles.

Figure 7: Coherency I: $(200)_{\operatorname{mat}} \|(\overline{4} 40)_{o x}$, [001 $]_{\text {mat }} \|[110]_{o x}$ : (a) HR-TEM image; (b) contrast enhanced FFT-IFFT image of (a); (c) FFT of HR-TEM image; (d) simulated FFT diffraction pattern of Coherency I; (e) stereogram of Coherency I.

Figure 8: Coherency II: (002) mat $\left\|(\overline{4} 40)_{o x},[110]_{\text {mat }}\right\|[110]_{o x}$ : (a) HR-TEM image; (b) contrast enhanced FFT-IFFT image of (a); (c) FFT of HR-TEM image; (d) simulated FFT diffraction pattern of Coherency II; (e) stereogram of Coherency II.

Figure 9: Axis-Parallel: [110 $]_{\text {mat }} \|[110]_{o x}$ : (a) HR-TEM image of the cubic-on-cubic relation (labeled as Axis-parallel I); (b) FFT of (a); (c) another HR-TEM image of the cubic-on-cubic relation; (d) contrast enhanced FFT-IFFT image of (c) showing misfit dislocations; (e) special axis-parallel relation with only one $\langle 111\rangle_{\text {mat }} \|\langle 111\rangle_{o x}$ (labeled as Axis-parallel II); (f) FFT of (e).

Figure 10: A large nanoparticle with random orientation: (a) HR-TEM image of a large nanoparticle (32nm); (b) magnification of the interface shown in (a); (c) electron diffraction pattern of the matrix in (a) showing the [110] zone; (d) FFT of the nanoparticle part of (a) showing diffraction spots from the matrix, the nanoparticle and Moiré fringes. 
Figures

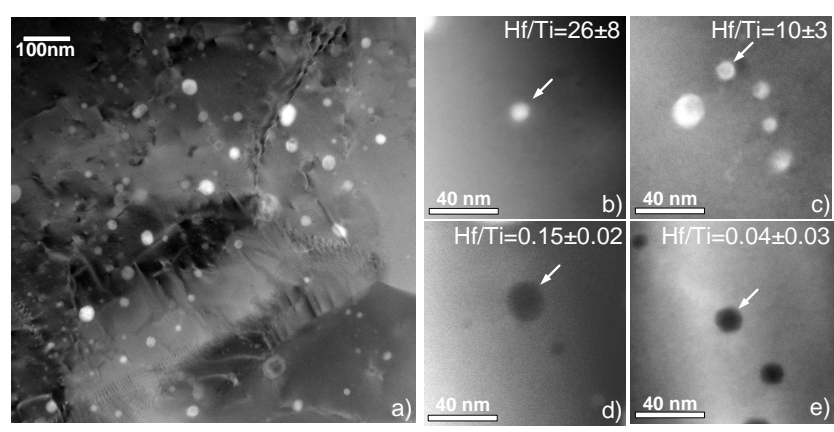

Figure 1: 


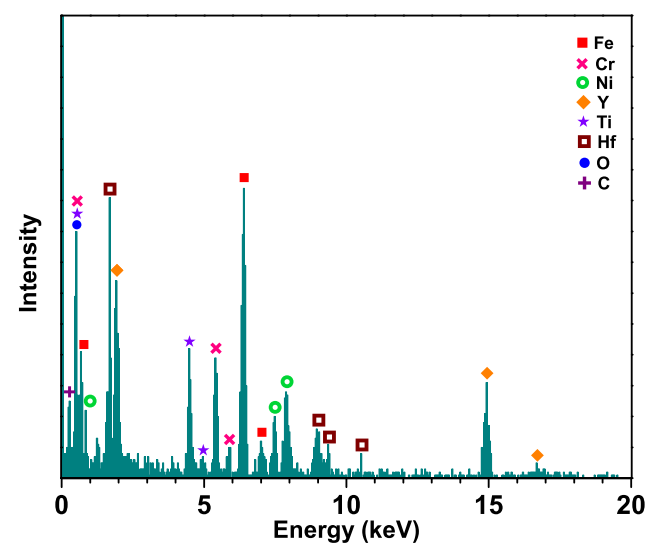

Figure 2: 


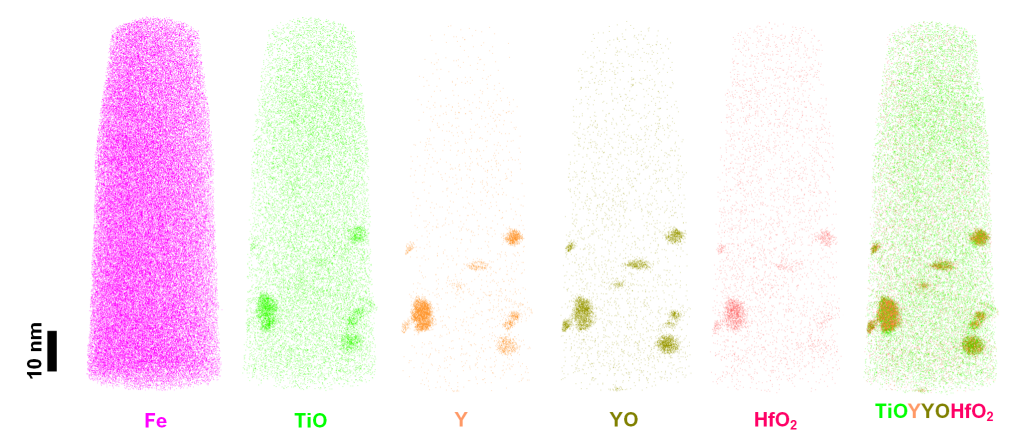

Figure 3: 


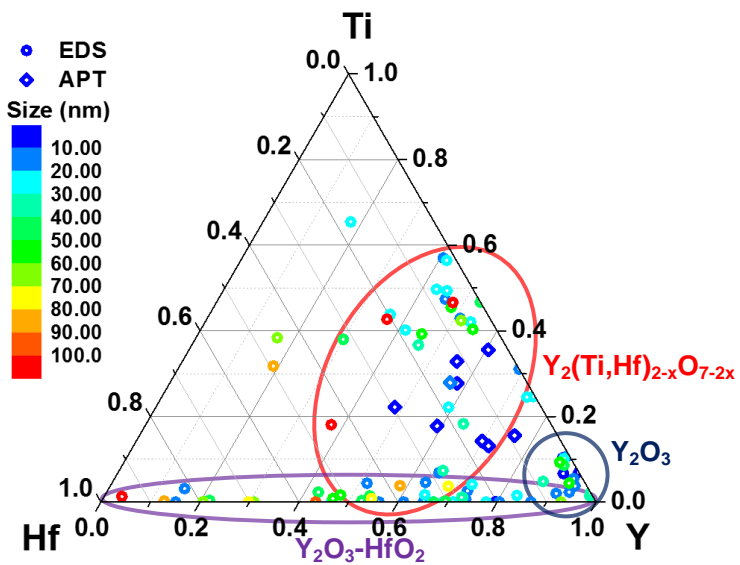

Figure 4: 


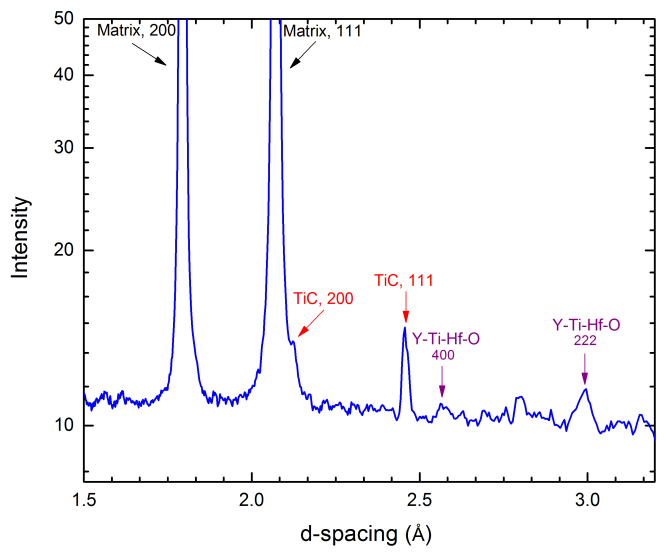

Figure 5: 

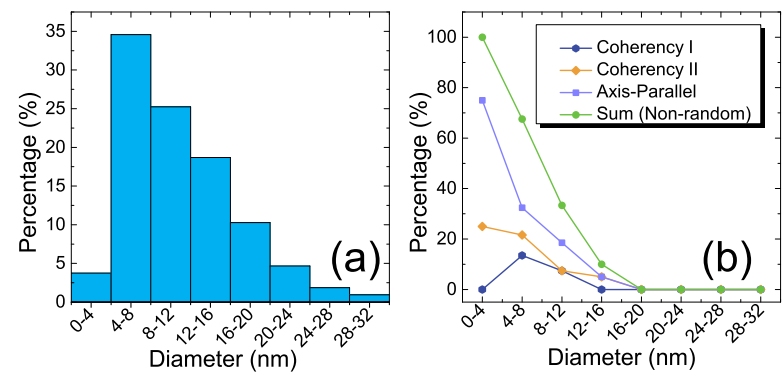

Figure 6: 


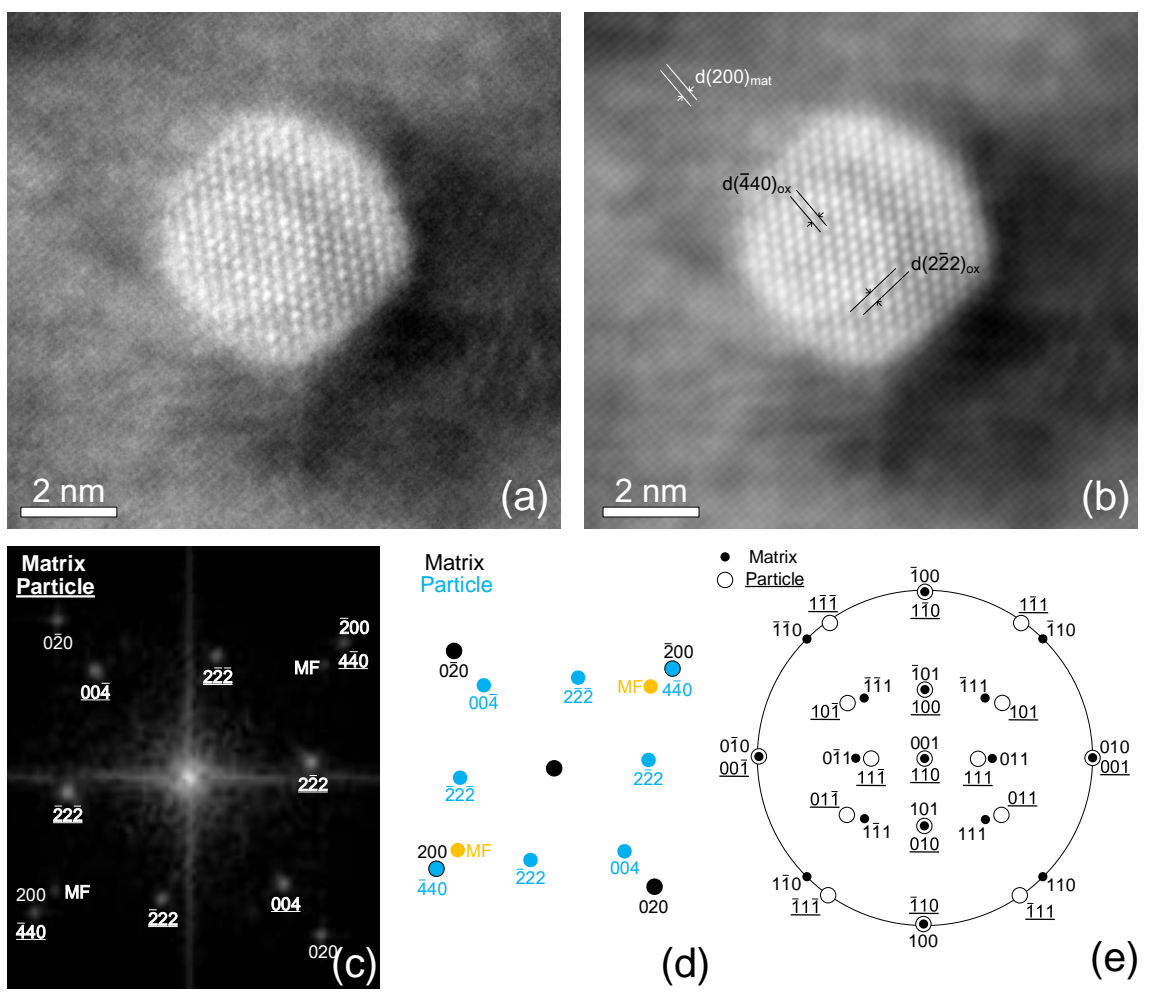

Figure 7: 


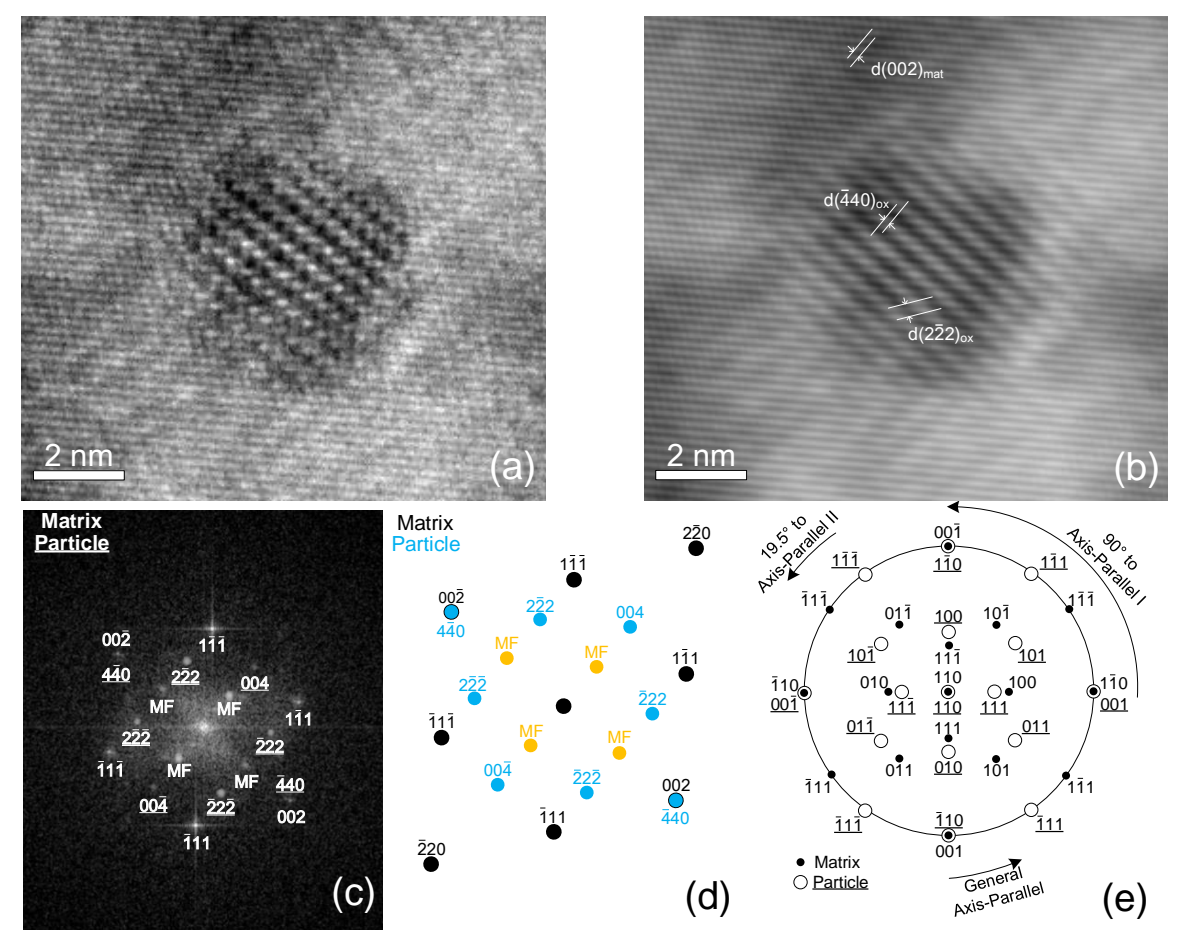

Figure 8: 


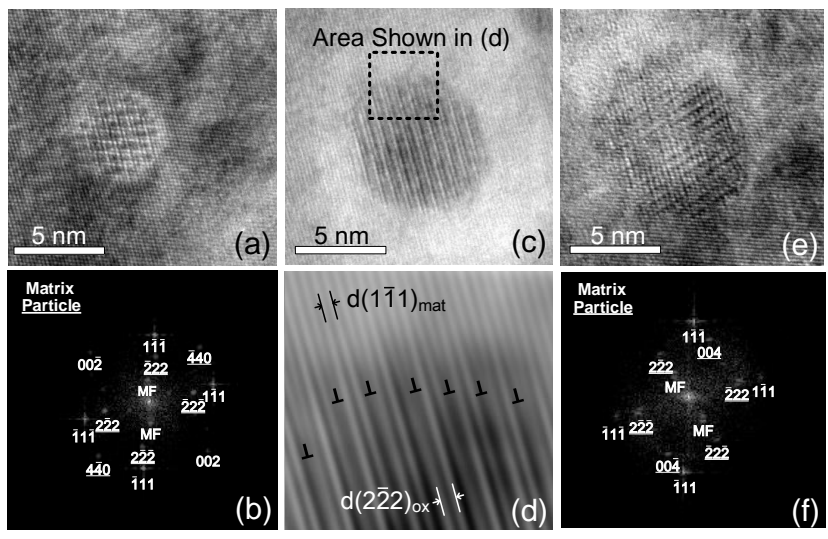

Figure 9: 


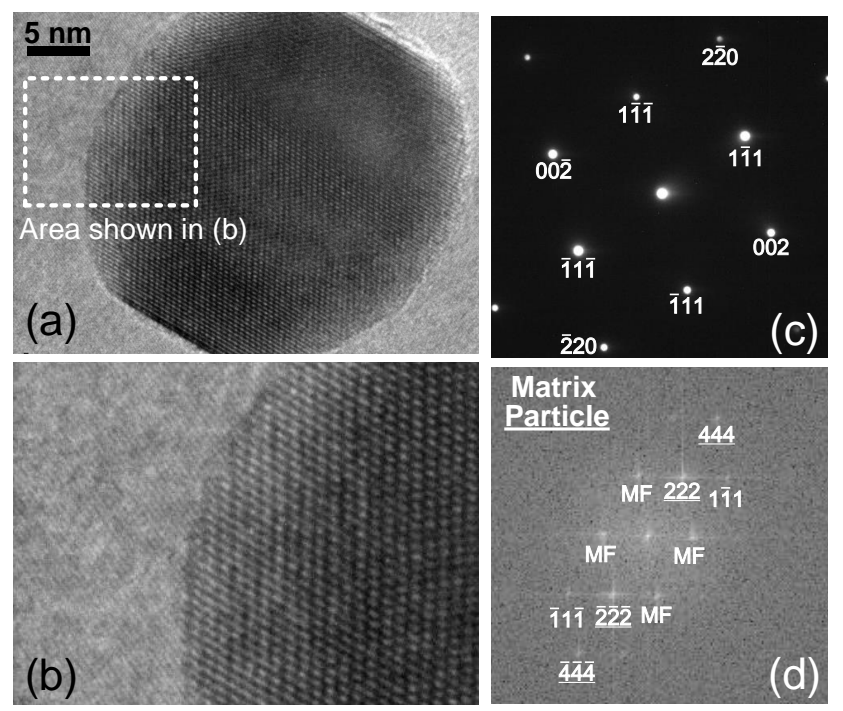

Figure 10: 